\title{
RIGIDITY OF EXTREMAL QUASIREGULARLY ELLIPTIC MANIFOLDS
}

\author{
RAMI LUISTO AND PEKKA PANKKA
}

\begin{abstract}
We show that for a closed $n$-manifold $N$ admitting a quasiregular mapping from Euclidean $n$-space the following are equivalent: (1) order of growth of $\pi_{1}(N)$ is $n,(2) N$ is aspherical, and (3) $\pi_{1}(N)$ is virtually $\mathbb{Z}^{n}$ and torsion free.
\end{abstract}

\section{INTRODUCTION}

In this note we consider closed Riemannian manifolds $N$ admitting a quasiregular mapping from $\mathbb{R}^{n}$, i.e. quasiregularly elliptic manifolds. A nonconstant continuous mapping $f: \mathbb{R}^{n} \rightarrow N$ is (K-)quasiregular if $f$ belongs to the Sobolev space $W_{\text {loc }}^{1, n}\left(\mathbb{R}^{n}, N\right)$ and satisfies the distortion inequality

$$
|D f|^{n} \leq K J_{f} \quad \text { a.e. in } \mathbb{R}^{n},
$$

where $D f$ is the differential of the map $f$ and $J_{f}$ the Jacobian determinant. By Reshetnyak's theorem, quasiregular mappings are branched covers, that is, discrete and open mappings, see e.g. [19, Theorem I.4.1].

In dimensions $n=2,3$, closed quasiregularly elliptic manifolds are fully understood. In dimension $n=2$, the manifolds are the 2-sphere $\mathbb{S}^{2}$ and the 2-torus $\mathbb{T}^{2}$ by the uniformization theorem and Stoilow factorization. In dimension $n=3$, the possible closed targets of quasiregular mappings from $\mathbb{R}^{3}$ are $\mathbb{S}^{3}, \mathbb{S}^{2} \times \mathbb{S}^{1}, \mathbb{T}^{3}$, and their quotients. The completeness of this list follows from the geometrization theorem; see Jormakka [15].

By Varopoulos' theorem [22, pp. 146-147] the fundamental group of a quasiregularly elliptic $n$-manifold, $n \geq 2$, has polynomial growth of order at most $n$. In particular, the fundamental group is virtually nilpotent by Gromov's theorem, see [10].

Our main theorem is the following characterization of quasiregularly elliptic manifolds which are extremal in the sense of Varopoulos' theorem. Recall that a manifold $N$ is aspherical if $\pi_{k}(N)=0$ for $k>1$. We denote the degree of polynomial growth of a finitely generated group $G$ by $\operatorname{ord}(G)$.

Theorem 1.1. Let $N$ be a closed quasiregularly elliptic n-manifold. Then the following are equivalent:

(1) $\operatorname{ord}\left(\pi_{1}(N)\right)=n$,

(2) $N$ is aspherical, and

(3) $\pi_{1}(N)$ is virtually $\mathbb{Z}^{n}$ and torsion free.

Date: October 16, 2018.

2010 Mathematics Subject Classification. $30 \mathrm{C65}$ (57M12).

The authors are supported by the Academy of Finland project \#256228. 
Given the classification of quasiregularly elliptic manifolds in dimensions $n=2,3$, we conclude that, in these dimensions, only manifolds $N$ satisfying (11) are quotients of the $n$-torus $\mathbb{T}^{n}$. For $n \geq 5$, by deep rigidity theorems of Hsiang and Wall [14 and Farrell and Hsiang [8] conditions (2) and (3) imply that $N$ is homeomorphic to a quotient of $\mathbb{T}^{n}$; see also Freedman and Quinn [9, Section 11.5] for the extension to $n=4$. Since the $n$-torus has a unique Lipschitz structure for $n \geq 5$ by Sullivan's theorem [20, Theorem 2], we have the following corollary.

Corollary 1.2. Let $N$ be a closed quasiregularly elliptic $n$-manifold, $n \geq 2$, with $\operatorname{ord}\left(\pi_{1}(N)\right)=n$. Then there exists a (topological) covering map $\mathbb{T}^{n} \rightarrow$ $N$. For $n \neq 4$, there exists a locally bilipschitz covering map $\mathbb{T}^{n} \rightarrow N$.

Theorem 1.1 can be viewed as a (branched) quasiconformal Bieberbach theorem. By the classical Bieberbach theorem, an n-dimensional crystallographic group $\Gamma$ (that is, a cocompact discrete group of Euclidean isometries of $\mathbb{R}^{n}$ ) is virtually $\mathbb{Z}^{n}$ and torsion free. By a classical result of Auslander and Kuranishi [1] all groups which are virtually $\mathbb{Z}^{n}$ and torsion free are crystallographic; see also Thurston [21, Section 4.2].

Corollary 1.3. Let $N$ be a closed orientable Riemannian $n$-manifold, $n \neq 4$, satisfying $\operatorname{ord}\left(\pi_{1}(N)\right)=n$. Then $N$ is quasiregularly elliptic if and only if $N$ is bilipschitz homeomorphic to $\mathbb{R}^{n} / \Gamma$, where $\Gamma$ is a crystallographic group.

In particular, we obtain a partial answer to a question of Bonk and Heinonen [3, p. 222].

Corollary 1.4. Let $N$ be a closed quasiregularly elliptic n-manifold satisfying $\operatorname{ord}\left(\pi_{1}(N)\right)=n$. Then

$$
\operatorname{dim} H^{*}(N ; \mathbb{Q}) \leq 2^{n} .
$$

Finally, Theorem 1.1 bears a close resemblance to a result of Gromov on elliptic manifolds [11, Corollary 2.43]: The fundamental group of a closed aspherical elliptic manifold is virtually $\mathbb{Z}^{n}$. A manifold $N$ is called elliptic if there exists a Lipschitz map $\mathbb{R}^{n} \rightarrow N$ of non-zero asymptotic degree. We refer to [11, Section 2.41] for a detailed discussion on elliptic manifolds. By Theorem 1.1, for quasiregularly elliptic manifolds, the topological assumption on asphericity can be replaced by the geometric assumption on the Euclidean volume growth of the universal cover.

1.1. Sketch of the proof of Theorem 1.1. The main content of Theorem 1.1 is that (11) implies (2). Well-known arguments in cohomological group theory show that in this setting (2) implies (3) since the growth rate of the fundamental group of a quasiregularly elliptic manifold is polynomial. Condition (3) trivially implies (11).

The rest of the paper is devoted to the proofs of implications (11) $\Rightarrow(2)$ and (2) $\Rightarrow(3)$. In Section 2 we discuss the observation that the universal cover $\tilde{N}$ of $N$ is a Loewner space in the sense of Heinonen and Koskela [13. The proof is based on the verification of a $(1, n)$-Poincaré inequality on $\tilde{N}$. Using a similar argument as in 17, we give a simple proof for a $(1,1)$-Poincaré inequality on $\tilde{N}$, which yields the required $(1, n)$-Poincaré 
inequality trivially; note that Saloff-Coste's $(2,2)$-Poincaré inequality for $\pi_{1}(N)$ in [17] also suffices.

Using the Euclidean volume growth and the Loewner property of $\tilde{N}$, we show that there exists a proper map $\mathbb{R}^{n} \rightarrow \tilde{N}$ and that $N$ is aspherical. Finally, in Section 4, we discuss the implication that asphericity of $N$ virtually detects the group $\pi_{1}(N)$. The argument is almost verbatim to the proof of Bridson and Gersten in [4, Theorem 5.9] for quasi-isometric rigidity of $\mathbb{Z}^{n}$, although our method allows us to detect the quasi-isometry type of $\pi_{1}(N)$ only posteriori.

Acknowledgements. We would like to thank the referees for suggestions and helpful remarks.

\section{The Loewner Property}

A metric measure space $(X, d, \mu)$ is $n$-Loewner if there exists a function $\phi:[0, \infty) \rightarrow[0, \infty)$ such that

$$
\bmod _{n}(E, F) \geq \phi(t)
$$

whenever $E$ and $F$ are two disjoint, nondegenerate continua in $X$ and

$$
t \geq \frac{\operatorname{dist}(E, F)}{\min (\operatorname{diam} E, \operatorname{diam} F)}
$$

Here $\bmod _{n}(E, F)$ is the $n$-modulus of the family $\Gamma(E, F)$ of all paths connecting $E$ and $F$, that is,

$$
\bmod _{n}(E, F)=\inf \int_{X} \rho^{n} \mathrm{~d} \mu,
$$

where the infimum is taken over all nonnegative Borel functions $\rho: X \rightarrow$ $[0, \infty]$ satisfying

$$
\int_{\gamma} \rho \mathrm{d} s \geq 1
$$

for all locally rectifiable paths $\gamma \in \Gamma(E, F)$.

In this section, we consider the Loewner property of universal covers $\tilde{N}$ of closed Riemannian manifolds $N$ satisfying ord $\left(\pi_{1}(N)\right)=n$. The Riemannian metric induced by the covering $\tilde{N} \rightarrow N$ makes $\tilde{N}$ into a geodesic metric space with Euclidean volume growth, that is, $\tilde{N}$ is Ahlfors $n$-regular:

$$
\mathcal{H}^{n}(B(x, r)) \approx r^{n}
$$

for every ball $B(x, r)$ in $\tilde{N}$ of radius $r>0$ about $x \in \tilde{N}$.

It seems that the following theorem has not been reported in the literature although it is well-known to the experts.

Theorem 2.1. Let $N$ be a closed and connected Riemannian n-manifold for $n \geq 2$ satisfying $\operatorname{ord}\left(\pi_{1}(N)\right)=n$. Then the universal cover $\tilde{N}$ of $N$ is $n$-Loewner.

Remark 1. Since $\tilde{N}$ is Ahlfors n-regular and geodesic, the Loewner property (2.1) holds with $\phi$ satisfying

$$
\phi(t) \approx \begin{cases}\log (t), & \text { when } t \text { is small } \\ \log (t)^{1-n}, & \text { when } t \text { is large }\end{cases}
$$


see [13, Theorem 3.6].

By a result of Heinonen and Koskela [13, Corollary 5.13], $\tilde{N}$ is $n$-Loewner if and only if $\tilde{N}$ supports a weak $(1, n)$-Poincaré inequality: there exists $C>0$ and $\lambda \geq 1$ for which

$$
f_{B(x, r)}\left|f-f_{B(x, r)}\right| \leq C r\left(f_{B(x, \lambda r)}|\nabla f|^{n}\right)^{\frac{1}{n}}
$$

for all $x \in \tilde{N}, r>0$ and $f \in C^{\infty}(\tilde{N})$. Here we denote by $f_{B(x, r)}$ the average

$$
f_{B(x, r)} f(y) \mathrm{d} y .
$$

The weak $(1, n)$-Poincaré inequality follows directly from Kleiner's weak $(2,2)$-Poincaré inequality in [17, Theorem 2.2.] by Hölder's inequality. Laurent Saloff-Coste's argument in [17] can, however, be used to show also that $\tilde{N}$ satisfies a weak $(1,1)$-Poincaré inequality. Since we have not seen this explicitly stated in the literature, we give a short proof based on [17, Theorem 2.2] and [6, p. 308-309] for the reader's convenience.

Theorem 2.2. Let $N$ be a closed Riemannian n-manifold with a polynomially growing fundamental group. Let $\tilde{N}$ be the universal cover of $N$. Then there exists $C>0$ so that

$$
f_{B(x, r)}\left|f-f_{B(x, r)}\right| \leq C r f_{B(x, 3 r)}|\nabla f|
$$

for all $x \in \tilde{N}, r>0$, and $f \in C^{\infty}(\tilde{N})$.

The proof is based on the following lemma. In the statement, we assume that we have chosen a fixed finite (symmetric) generating set $S$ for a group $\Gamma$, and denote by

$$
B(x, r)=\left\{x s_{1} \cdots s_{r} \in \Gamma: s_{i} \in S \text { for } i=1, \ldots, r\right\}
$$

the ball of radius $r>0$ about $x$ in $\Gamma, V(r)=\# B(e, r)$,

$$
f_{B(x, r)}:=\frac{1}{V(r)} \sum_{y \in B(x, r)} f(y) \quad \text { and } \quad \nabla f(y):=\sum_{z \in y S}|f(z)-f(y)| .
$$

Lemma 2.3. Let $\Gamma$ be a finitely generated group. Then

$$
\sum_{y \in B(x, r)}\left|f(y)-f_{B(x, r)}\right| \leq(r+1) \frac{V(2 r)}{V(r)} \sum_{y \in B(x, 3 r)}|\nabla f(y)|
$$

for all $r>0, x \in \Gamma$, and $f: \Gamma \rightarrow \mathbb{R}$.

Proof. We fix for each $g \in G$ a geodesic $\gamma_{g}:\{0, \ldots,|g|\} \rightarrow \Gamma$ in $\Gamma$ connecting the neutral element $e$ of $\Gamma$ to $g$.

For any $r>0$ and $y \in B(x, r)$ the mapping

$$
B(x, r) \times\{0, \ldots,|y|\} \rightarrow G, \quad(x, i) \mapsto x \gamma_{y}(i),
$$

is at most $(r+1)$-to-1. 
For every $y_{0} \in B(x, r)$, we have

$$
\begin{aligned}
V(r) & \sum_{y \in B(x, r)}\left|f(y)-f_{B(x, r)}(x)\right| \leq \sum_{y, z \in B(x, r)}|f(y)-f(z)| \\
\leq & \sum_{y \in B(x, r)} \sum_{w \in B(x, 2 r)}|f(y w)-f(z)| \\
\leq & \sum_{y \in B(x, r)} \sum_{w \in B(x, 2 r)} \sum_{i=0}^{\ell\left(\gamma_{w}\right)-1}\left|f\left(y \gamma_{w}^{i+1}\right)-f\left(y \gamma_{w}^{i}\right)\right| \\
\leq & \sum_{w \in B(x, 2 r)} \sum_{i=0}^{\ell\left(\gamma_{w}\right)-1}\left|\nabla f\left(y_{0} \gamma_{w}^{i}\right)\right| .
\end{aligned}
$$

On the other hand,

$$
\begin{aligned}
\sum_{w \in B(x, 2 r)} \sum_{i=0}^{\ell\left(\gamma_{w}\right)}\left|\nabla f\left(y \gamma_{w}^{i}\right)\right| & \leq \sum_{w \in B(x, 2 r)} \sum_{z \in B(x, 3 r)}(r+1)|\nabla f(z)| \\
& \leq V(2 r)(r+1) \sum_{z \in B(x, 3 r)}|\nabla f(z)| .
\end{aligned}
$$

This proves the claim.

Proof of Theorem 2.2. For every $r>0$, the universal cover $\tilde{N}$ satisfies the local $(1,1)$-Poincaré inequality

$$
\int_{B(x, r)}\left|f(y)-f_{B(x, r)}\right| d y \leq \beta \int_{B(x, r)}|\nabla f|
$$

for $f \in C^{\infty}(\tilde{N})$, where $\beta$ depends on $r$; see Kanai [16, Lemma 8].

Since $\pi_{1}(N)$ has polynomial growth, the ratio $V(2 r) / V(r)$ is uniformly bounded for all $x \in \pi_{1}(N)$ and $r>1$. Thus, by Lemma 2.3, there exists $C>1$ so that

$$
\sum_{y \in B(x, r)}\left|f(y)-f_{B(x, r)}\right| \leq C r \sum_{y \in B(x, 3 r)}|\nabla f(y)|
$$

for all $r>0, x \in \Gamma$ and $f: \Gamma \rightarrow \mathbb{R}$.

The claim now follows from [7, Théorème 7.2.(3)].

This concludes the proof of Theorem 2.1.

\section{EUCLIDEAN VOLUME GROWTH AND ASPHERICITY}

In this section we show that a closed quasiregularly elliptic manifold with maximally growing fundamental group is aspherical. We obtain this result by combining the following lemmas.

Lemma 3.1. Suppose $N$ is a closed quasiregularly elliptic $n$-manifold, $n \geq$ 2 , with $\operatorname{ord}\left(\pi_{1}(N)\right)=n$. Then there exists a proper quasiregular map $\mathbb{R}^{n} \rightarrow$ $\tilde{N}$ into the universal cover $\tilde{N}$ of $N$. 
Proof. By Zalcman's lemma (see Bonk-Heinonen [3, Corollary 2.2]), there exists a uniformly locally Hölder continuous quasiregular map $f: \mathbb{R}^{n} \rightarrow N$ satisfying

$$
\int_{B^{n}(x, r)} J_{f} \leq C r^{n}
$$

where $C>0$ is a constant independent of the ball $B^{n}(x, r) \subset \mathbb{R}^{n}$. Let $\tilde{f}$ be a lift of $f$ to the universal cover $\tilde{N}$ of $N$. Since the universal covering map is a local isometry, $\tilde{f}$ again satisfies inequality (3.1).

Since $\tilde{N}$ is Ahlfors $n$-regular and $n$-Loewner, we have that, for every $a \in \tilde{N}, d(\tilde{f}(x), a) \rightarrow \infty$ as $|x| \rightarrow \infty$ by the Onninen-Rajala theorem [18, Theorem 12.1]. Indeed, (a) in [18, Theorem 12.1] can be replaced with (3.1); see Lemma 12.13 and (12.3) in [18. Thus $\tilde{f}$ is a proper map.

Lemma 3.2. Let $N$ be a connected, simply connected, and oriented Riemannian n-manifold. Then $N$ is aspherical if there exists a proper branched cover $\mathbb{R}^{n} \rightarrow \tilde{N}$ into the universal cover $\tilde{N}$ of $N$.

Proof. Suppose there exists $k \geq 2$ for which $\pi_{k}(\tilde{N}) \neq 0$. Let $k \geq 2$ be the smallest such index. Then, by the Hurewicz isomorphism theorem, $\pi_{k}(\tilde{N})$ is isomorphic to $H_{k}(\tilde{N})$.

If $H_{k}(\tilde{N})$ is free abelian, the universal coefficient theorem for cohomology immediately yields $H^{k}(\tilde{N}, \mathbb{Z}) \neq 0$. If $H_{k}(\tilde{N})$ is not free, $\operatorname{Ext}\left(H_{k}(\tilde{N}), \mathbb{Z}\right) \neq 0$. Thus $H^{k+1}(\tilde{N}, \mathbb{Z}) \neq 0$ by the universal coefficient theorem.

We conclude that there exists an index $\ell \geq 2$ such that $H^{\ell}(\tilde{N}, \mathbb{Z}) \neq$ 0 . Since $N$ receives a proper branched cover from $\mathbb{R}^{n}$ the fundamental group $\pi_{1}(N)$ must be infinite. Thus the universal cover $\tilde{N}$ is unbounded, so $H_{c}^{0}(\tilde{N}, \mathbb{Z})=0$ and by the Poincaré duality $H^{n}(\tilde{N}, \mathbb{Z})=0$. In particular, $\ell<n$.

Let $f: \mathbb{R}^{n} \rightarrow \tilde{N}$ be a proper branched cover. Then $f^{*}: H_{c}^{n}(\tilde{N} ; \mathbb{Z}) \rightarrow$ $H_{c}^{n}\left(\mathbb{R}^{n} ; \mathbb{Z}\right)$ is non-trivial.

By the Poincaré duality, there exists $c \in H^{\ell}(\tilde{N}, \mathbb{Z})$ and $c^{\prime} \in H_{c}^{n-\ell}(\tilde{N}, \mathbb{Z})$ satisfying $c \cup c^{\prime} \neq 0 \in H_{c}^{n}(\tilde{N}, \mathbb{Z})$. Then

$$
f^{*} c \cup f^{*} c^{\prime}=f^{*}\left(c \cup c^{\prime}\right) \neq 0 \in H_{c}^{n}\left(\mathbb{R}^{n}, \mathbb{Z}\right)
$$

This is a contradiction, since $f^{*} c=0$. Thus $\pi_{k}(N)=0$ for all $k \geq 2$.

\section{Closed aspherical QUASiregularly Elliptic Manifolds}

In this section we show that (2) implies (3) in the setting of Theorem 1.1. see also [11, Corollary 2.43]. The argument of the proof is almost identical to the proof of theorem [4, Theorem 5.9.]: a finitely generated group that is quasi-isometric to the group $\mathbb{Z}^{n}$ is virtually $\mathbb{Z}^{n}$. Due to the differences in the setting, we give a proof for the reader's convenience.

Lemma 4.1. Let $N$ be a closed, connected, aspherical $n$-manifold with $\operatorname{ord}\left(\pi_{1}(N)\right) \leq n$. Then $\pi_{1}(N)$ is virtually $\mathbb{Z}^{n}$ and torsion free.

Proof. Since $N$ is aspherical, the cohomological dimension $\operatorname{cd}\left(\pi_{1}(N)\right)$ of $\pi_{1}(N)$ is at most $n$ by [5, Prop VIII.2.2]. Thus $\pi_{1}(N)$ has finite cohomological dimension and is torsion free by [5, Prop VIII.2.8]. 
Since $\pi_{1}(N)$ has polynomial growth, it is virtually nilpotent. Let $\hat{N}$ be a finite cover of $N$ having nilpotent fundamental group. Then $\pi_{1}(\hat{N})$ has a lower central series

$$
\pi_{1}(\hat{N})=\Gamma_{0} \supset \Gamma_{1} \supset \cdots \supset \Gamma_{m}=\{1\}
$$

with free abelian quotients $\Gamma_{k-1} / \Gamma_{k}$ for $k=1, \ldots, m$. Moreover, since $\pi_{1}(\hat{N})$ is finitely generated, the cohomological dimension coincides with the Hirsh number of $\pi_{1}(\hat{N})$, that is,

$$
\operatorname{cd}\left(\pi_{1}(\hat{N})\right)=\sum_{k=1}^{m} \operatorname{rank}\left(\Gamma_{k-1} / \Gamma_{k}\right) .
$$

We refer to [12, Section 8.8] (especially Theorem 5) for these details.

On the other hand, by the growth formula for nilpotent groups (see Bass [2]), we have

$$
\operatorname{ord}\left(\pi_{1}(\hat{N})\right)=\sum_{k=1}^{m} k \operatorname{rank}\left(\Gamma_{k-1} / \Gamma_{k}\right) .
$$

Since $\operatorname{cd}(\hat{N})=n$ by [5, Theorem VIII.8.1], we have

$$
\sum_{k=1}^{m} k \operatorname{rank}\left(\Gamma_{k-1} / \Gamma_{k}\right)=\operatorname{ord}\left(\pi_{1}(\hat{N})\right) \leq \operatorname{cd}\left(\pi_{1}(\hat{N})\right)=\sum_{k=1}^{m} \operatorname{rank}\left(\Gamma_{k-1} / \Gamma_{k}\right)
$$

by combining (4.1), (4.2), and (4.3). Thus

$$
\operatorname{rank}\left(\Gamma_{k-1} / \Gamma_{k}\right)=0
$$

for all $k=2, \ldots, m$ and $\pi_{1}(\hat{N})$ is abelian. Thus $\pi_{1}(N)$ is virtually $\mathbb{Z}^{n}$.

\section{REFERENCES}

[1] L. Auslander and M. Kuranishi, On the holonomy group of locally Euclidean spaces, Ann. of Math. (2) 65 (1957), 411-415. MR 0086341 (19,168b)

[2] H. Bass, The degree of polynomial growth of finitely generated nilpotent groups, Proc. London Math. Soc. (3) 25 (1972), 603-614. MR 0379672 (52 \#577)

[3] Mario Bonk and Juha Heinonen, Quasiregular mappings and cohomology, Acta Math. 186 (2001), no. 2, 219-238. MR 2002e:30021

[4] M. R. Bridson and S. M. Gersten, The optimal isoperimetric inequality for torus bundles over the circle, Quart. J. Math. Oxford Ser. (2) 47 (1996), no. 185, 1-23. MR 1380947 (97c:20047)

[5] Kenneth S. Brown, Cohomology of groups, Graduate Texts in Mathematics, vol. 87, Springer-Verlag, New York, 1994, Corrected reprint of the 1982 original. MR 1324339 (96a:20072)

[6] Thierry Coulhon and Laurent Saloff-Coste, Isopérimétrie pour les groupes et les variétés, Rev. Mat. Iberoamericana 9 (1993), no. 2, 293-314. MR 1232845 (94g:58263)

[7] _ Variétés riemanniennes isométriques à l'infini, Rev. Mat. Iberoamericana 11 (1995), no. 3, 687-726. MR 1363211 (96m:53035)

[8] F. T. Farrell and W. C. Hsiang, Topological characterization of flat and almost flat Riemannian manifolds $M^{n}(n \neq 3,4)$, Amer. J. Math. 105 (1983), no. 3, 641-672. MR 704219 (84k:57017)

[9] Michael H. Freedman and Frank Quinn, Topology of 4-manifolds, Princeton Mathematical Series, vol. 39, Princeton University Press, Princeton, NJ, 1990. MR 1201584 (94b:57021)

[10] Mikhael Gromov, Groups of polynomial growth and expanding maps, Inst. Hautes Études Sci. Publ. Math. (1981), no. 53, 53-73. MR 623534 (83b:53041) 
[11] Misha Gromov, Metric structures for Riemannian and non-Riemannian spaces, Progress in Mathematics, vol. 152, Birkhäuser Boston Inc., Boston, MA, 1999. MR 2000d:53065

[12] Karl W. Gruenberg, Cohomological topics in group theory, Lecture Notes in Mathematics, Vol. 143, Springer-Verlag, Berlin, 1970. MR 0279200 (43 \#4923)

[13] Juha Heinonen and Pekka Koskela, Quasiconformal maps in metric spaces with controlled geometry, Acta Math. 181 (1998), no. 1, 1-61. MR 1654771 (99j:30025)

[14] W.-C. Hsiang and C. T. C. Wall, On homotopy tori. II, Bull. London Math. Soc. 1 (1969), 341-342. MR 0258044 (41 \#2691)

[15] Jorma Jormakka, The existence of quasiregular mappings from $\mathbf{R}^{3}$ to closed orientable 3-manifolds, Ann. Acad. Sci. Fenn. Ser. A I Math. Dissertationes (1988), no. 69, 44. MR MR973719 (90b:57005)

[16] Masahiko Kanai, Rough isometries and the parabolicity of Riemannian manifolds, J. Math. Soc. Japan 38 (1986), no. 2, 227-238. MR 833199 (87e:53066)

[17] Bruce Kleiner, A new proof of Gromov's theorem on groups of polynomial growth, J. Amer. Math. Soc. 23 (2010), no. 3, 815-829. MR 2629989 (2012d:20089)

[18] Jani Onninen and Kai Rajala, Quasiregular mappings to generalized manifolds, J. Anal. Math. 109 (2009), 33-79. MR 2585391 (2011c:30063)

[19] Seppo Rickman, Quasiregular mappings, Ergebnisse der Mathematik und ihrer Grenzgebiete (3) [Results in Mathematics and Related Areas (3)], vol. 26, Springer-Verlag, Berlin, 1993. MR 95g:30026

[20] Dennis Sullivan, Hyperbolic geometry and homeomorphisms, Geometric topology (Proc. Georgia Topology Conf., Athens, Ga., 1977), Academic Press, New York, 1979, pp. 543-555. MR 537749 (81m:57012)

[21] William P. Thurston, Three-dimensional geometry and topology. Vol. 1, Princeton Mathematical Series, vol. 35, Princeton University Press, Princeton, NJ, 1997, Edited by Silvio Levy. MR 1435975 (97m:57016)

[22] N. Th. Varopoulos, L. Saloff-Coste, and T. Coulhon, Analysis and geometry on groups, Cambridge Tracts in Mathematics, vol. 100, Cambridge University Press, Cambridge, 1992. MR 95f:43008

Department of Mathematics and Statistics, P.O. Box 68 (Gustaf Hällströmin Katu 2B), FI-00014 University of Helsinki, Finland

Department of Mathematics and Statistics, P.O. Box 68 (Gustaf Hällströmin katu 2B), FI-00014 University of Helsinki, Finland and Department of Mathematics and Statistics, P.O. Box 35, FI-40014 University of Jyväskylä, Finland 